\title{
Genome sequences published outside of Standards in Genomic Sciences, May-June 2012
}

\author{
Oranmiyan W. Nelson ${ }^{1}$ and George M. Garrity ${ }^{1}$ \\ ${ }^{1}$ Editorial Office, Standards in Genomic Sciences and Department of Microbiology, \\ Michigan State University, East Lansing, MI, USA
}

\begin{abstract}
The purpose of this table is to provide the community with a citable record of publications of ongoing genome sequencing projects that have led to a publication in the scientific literature. While our goal is to make the list complete, there is no guarantee that we may have omitted one or more publications appearing in this time frame. Readers and authors who wish to have publications added to subsequent versions of this list are invited to provide the bibliographic data for such references to the SIGS editorial office.
\end{abstract}

\section{Domain Archaea}

Phylum Euryarchaeota

Thermococcus zilligii AN1, sequence accession AJLF00000000 [1]

\section{Domain Bacteria}

Phylum Deinococcus-Thermus

Thermus sp. Strain RL, sequence accession AIJQ00000000 [2]

Phylum Proteobacteria

Azospira suillum Strain PS, sequence accession CP003153 [3]

Bradyrhizobium elkanii 587, sequence accession AJJK00000000 [4]

Burkholderia sp. strain SJ98, sequence accession AJHK00000000 [5]

Corallococcus coralloides DSM 2259, sequence accession CP003389 [6]

Diplorickettsia massiliensis strain 20B, sequence accession AJGC00000000 [7]

Escherichia coli DECA collection, AIEV00000000 through AIEZ00000000, AIFA00000000 through AIFZ00000000, AIGA00000000 through AIGZ00000000, AIHA00000000 through AIHS00000000 [8]

Escherichia coli 0157:H7 strain EDL933, sequence accession NC_002655.2 [9]

Escherichia coli J53, sequence accession AICK00000000 [10]
Escherichia coli Strain NCCP15657, sequence accession AJLU00000000 [11]

Escherichia coli NCCP15647, sequence accession AJMB00000000 [12]

Escherichia coli NCCP15658, sequence accession AJMD00000000 [13]

Francisella philomiragia ATCC 2501, sequence accession NC_010336.1 [14]

Gammaproteobacterial BDW918, sequence accession AJMK00000000 [15]

Glaciecola punicea ACAM 611 ${ }^{\mathrm{T}}$, sequence accession BAET00000000 [16]

Halomonas sp. Strain KM-1, sequence accession BAEU01000001 through BAEU01000173 [9]Vibrio sp. Strain EJY3, sequence accession CP003241 and CP003242 [17]

Helicobacter cinaedi Strain PAGU611, sequence accession AP012344 and AP012345 [18]

Helicobacter pylori hpEurope Strain N6, sequence accession CAHX01000001 through CAHX01000054 [19]

Kingella kingae septic arthritis isolate PYKK081, sequence accession AJGB00000000 [20]

Klebsiella oxytoca 11492-1, sequence accession AIEM01000000 [21]

Klebsiella pneumoniae strain KCTC 2242, sequence accession CP002910 and CP002911 [22]

Klebsiella pneumoniae Strain LCT-KP214, sequence accession AJHE00000000 [23] 
Marinobacter hydrocarbonoclasticus SP17, sequence accession FO203363 [24]

Pantoea sp. Strain Sc 1, sequence accession AJFP00000000 [25]

Pasteurella multocida HN06, sequence accession CP003313 (chromosome) and CP003314 (plasmid) [26]

Phaeospirillum molischianum DSM120, sequence accession CAHP01000001 through CAHP01000061 [27]

Providencia stuartii Clinical Isolate MRSN 2154, sequence accession CP003488 [28]

Pseudoalteromonas arctica A 37-1-2, sequence accession AHBY00000000 [29]

Pseudoalteromonas citrea NCIMB 1889, sequence accession AHBZ00000000 [29]

Pseudoalteromonas flavipulchra JG1, sequence accession AJMP00000000 [29]

Pseudoalteromonas marina mano4, sequence accession AHCB00000000 [29]

Pseudoalteromonas piscicida JCM 20779, sequence accession AHCC00000000 [29]

Pseudoalteromonas spongiae UST010723-006, sequence accession AHCE00000000 [29]

Pseudoalteromonas undina NCIMB 2128, sequence accession AHCF00000000 [29]

Pseudoalteromonas haloplanktis ATCC 14393, sequence accession AHCA00000000 [29]

Pseudomonas aeruginosa Strain ATCC 27853, sequence accession AJKG00000000 [30]

Pseudomonas fragi Strains A22, sequence accession AHZY01000001 through AHZY01000114 [31]

Pseudomonas fragi Strains B25, sequence accession AHZX01000001 through AHZX01000227 [31]

Pseudomonas fuscovaginae, sequence accession AIEU00000000 [32]

Pseudomonas geniculata N1, sequence accession AJLO00000000 [33]

Pseudomonas mandelii Strain JR-1, sequence accession AJFM00000000.1 [34]

Pseudomonas sp. strain R62, sequence accession AHZM00000000 [35]

Pseudomonas sp. strain R81, sequence accession AHZN00000000 [35]
Pseudomonas syringae pathovar syringae strain FF5, sequence accession ACXZ00000000 [36]

Rahnella aquatilis CIP 78.65, sequence accession CP003244 (chromosome), CP003245 (plasmid pRahaq201), CP003246 (plasmid pRahaq202), CP003247 (plasmid pRahaq203) [37]

Rickettsia conorii subsp. indica, sequence accession AJHC00000000 [38]

Rubrivivax gelatinosus CBS, sequence accession AJFF00000000 [39]

Rubrivivax gelatinosus IL144, sequence accession AP012320 [40]

Salmonella enterica serovar Heidelberg strain 41563, sequence accession AJGX00000000 [41]

Salmonella enterica serovar Heidelberg strain 41565, sequence accession AJHA00000000 [41]

Salmonella enterica serovar Heidelberg strain 41566, sequence accession AJGZ00000000 [41]

Salmonella enterica serovar Heidelberg strain 41573, sequence accession AJGY00000000 [41]

Salmonella enterica serovar Heidelberg strain 41579, sequence accession AJGW00000000 [41]

Salmonella enterica subsp. enterica Serotype Heidelberg Strain B182, sequence accession CP003416 (chromosome) CP003417 (plasmid) [41]

Serratia sp. Strain M24T3, sequence accession AJHJ00000000 [42]

Shigella flexneri serotype 5a strain $\mathrm{M}^{\mathrm{T}} \mathrm{Sm}$, sequence accession AGNM00000000 [43]

Sphingomonas sp. strain PAMC 26621, sequence accession AIDW00000000 [44]

Sphingomonas sp., sequence accession AHHA00000000 [45]

Sphingomonas wittichii DP58, sequence accession AHKO00000000 [46]

Stenotrophomonas maltophilia D457, sequence accession HE798556 [47]

Vibrio campbellii PEL22A, sequence accession AHYY00000000 [48]

Vibrio cholerae 01, sequence accession CP003330 and CP003331 [49]

Vibrio cholerae strain IEC224, sequence accession CP003330 (chromosome I), CP003331 (chromosome II) [50] 
Xanthomonas citri pv. mangiferaeindicae strain LMG 941, sequence accession CAHO01000001 through CAHO01000195 [51]

\section{Phylum Firmicutes}

Bacillus cereus strain LCT-BC244, sequence accession AJGQ00000000 [52]

Bacillus cereus bacteriophage PBC1, sequence accession JQ619704 [52]

Bacillus licheniformis WX-02, sequence accession AHIF00000000 [53]

Bacillus methanolicus MGA3, sequence accession ADWW00000000 [54]

Bacillus methanolicus PB1, sequence accession AFEU00000000 [55]

Bacillus sp. strain JS, sequence accession CP003492 [56]

Bacillus sp. strain 5B6, sequence accession AJST00000000 [57]

Bifidobacterium breve CECT 7263, sequence accession AFVV00000000 [58]

Clostridium arbusti SL206 ${ }^{\mathrm{T}}$, sequence accession BAEV00000000 [59]

Clostridium thermocellum Wild-Type Strain YS, sequence accession AJGT00000000 [60]

Clostridium thermocellum AD2, sequence accession AJGS00000000 [61]

Enterococcus faecium strain LCT-EF90, sequence accession AJKH00000000 [62]

Lactobacillus salivarius SMXD51, sequence accession AICL00000000 [63]

Lactobacillus vini Strains LMG 23202T, sequence accession AHYZ00000000 [64]

Lactobacillus vini Strains JP7.8.9, sequence accession AHZA00000000 [65]

Listeria monocytogenes 07PF0776, sequence accession CP003414 [66]

Melissococcus plutonius DAT561, sequence accession AP012282 and AP012283 [67]

Paenibacillus mucilaginosus 3016, sequence accession CP003235 [68]

Rahnella aquatilis CIP 78.65, sequence accession [69]
Sporolactobacillus vineae SL153T, sequence accession BAEY01000001 through BAEY01000092 [70]

Staphylococcus aureus 118 (ST772), sequence accession AJGE00000000 [71]

Staphylococcus aureus C160, sequence accession ACUV00000000 [72]

Staphylococcus aureus C427, sequence accession ACSQ00000000 [72]

Streptococcus pneumoniae Strain ST556, sequence accession CP003357 [73]

Streptococcus mutans Strain LJ23, sequence accession AP012336 [91]

\section{Phylum Actinobacteria}

Blastococcus saxobsidens DD2, sequence accession F0117623 [74]

Corynebacterium diphtheriae bv. mitis NCTC 3529, sequence accession AJGI00000000 [75]

Corynebacterium pseudotuberculosis strain Cp267, sequence accession CP003407 [76]

Microbacterium laevaniformans Strain OR221, sequence accession AJGR00000000 [77]

Mycobacterium abscessus subsp. bolletii $\mathrm{BD}^{\mathrm{T}}$, sequence accession AHAS00000000 [78]

Mycobacterium abscessus strain M93, sequence accession AJGF00000000 [79]

Mycobacterium intracellulare clinical strain MOTT02, sequence accession CP003323 [80]

Mycobacterium intracellulare clinical strain MOTT64, sequence accession CP003324 [81]

Mycobacterium intracellulare Strain ATCC $13950^{\mathrm{T}}$, sequence accession CP003322 [82]

Mycobacterium phlei Type Strain RIVM601174, sequence accession AJFJ00000000 [83]

Mycobacterium tuberculosis Erdman, sequence accession AP012340 [84]

Mycobacterium xenopi Type Strain RIVM700367, sequence accession AJFI00000000 [85]

Nocardia brasiliensis HUJEG-1, sequence accession AIHV00000000 [86]

Propionibacterium acnes strain PRP-38, sequence accession AIJP00000000 [87]

Strain IMCC13023, sequence accession AJKR00000000 [88] 
Rhodococcus sp. Strain P14, sequence accession AJFC00000000 [89]

Rhodococcus imtechensis RKJ300, sequence accession AJJH00000000 [90]

Streptomyces somaliensis DSM 40738, sequence accession AJJM00000000 [92]

Phylum Planctomycetes

Schlesneria paludicola, sequence accession AHZR00000000 [93]

Singulisphaera acidiphila, sequence accession AHZQ00000000 [93]

Zavarzinella formosa, sequence accession AIAB00000000 [93]

\section{Phylum Spirochaetes}

Borrelia crocidurae, sequence accession CP003426

(chromosome), CP003427 through CP003465 (plasmids) [94]

\section{Phylum Bacteroidetes}

Aquimarina agarilytica ZC1, sequence accession AHHE00000000 [95]

Fibrella aestuarina BUZ $2^{\mathrm{T}}$, sequence accession HE796683 (chromosome) and HE796684 (plasmid) [96]

\section{References}

1. Kim BK, Lee SH, Kim SY, Jeong H, Kwon SK, Lee $\mathrm{CH}$, Song JY, Yu DS, Kang SG, Kim JF. Genome Sequence of an Oligohaline Hyperthermophilic Archaeon, Thermococcus zilligii AN1, Isolated from a Terrestrial Geothermal Freshwater Spring. I Bacteriol 2012; 194:3765-3766. PubMed http://dx.doi.org/10.1128//B.00655-12

2. Dwivedi V, Sangwan N, Nigam A, Garg N, Niharika N, Khurana P, Khurana JP, Lal R. Draft Genome Sequence of Thermus sp. Strain RL, Isolated from a Hot Water Spring Located atop the Himalayan Ranges at Manikaran, India. J

Bacteriol 2012; 194:3534. PubMed http://dx.doi.org/10.1128//B.00604-12

3. Byrne-Bailey KG, Coates JD. Complete Genome Sequence of the Anaerobic Perchlorate-Reducing Bacterium Azospira suillum Strain PS. J Bacteriol 2012; 194:2767-2768. PubMed http://dx.doi.org/10.1128//B.00124-12

4. de Souza JAM, Tieppo E, Magnani GS, Alves LMC, Cardoso RL, Cruz LM, de Oliveira LF, Raittz RT, de Souza EM, Pedrosa FO, et al. Draft Ge-
Flavobacterium columnare ATCC 49512, sequence accession CP003222 [97]

Flavobacterium indicum GPSTA100-9T, sequence accession HE774682 [98]

Gillisia sp. strain CBA3202, sequence accession AJLT00000000 [99]

Imtechella halotolerans $\mathrm{K} 1^{\mathrm{T}}$, sequence accession AJJU00000000 [100]

Myroides injenensis M09-0166 ${ }^{\mathrm{T}}$, sequence accession [101]

Marinilabilia salmonicolor JCM 21150 ${ }^{\mathrm{T}}$, sequence accession AJKI00000000 [102]

Pedobacter agri PB92 ${ }^{\mathrm{T}}$, sequence accession AJLG00000000 [103]

Riemerella anatipestifer, sequence accession CP003388 [104]

Phylum Verrucomicrobia

Methylacidiphilum fumariolicum strain SolV, sequence accession CAHT01000001 through CAHT01000109 [105]

Opitutaceae bacterium Strain TAV1, sequence accession AHKS00000000 [106]

nome Sequence of the Nitrogen-Fixing Symbiotic Bacterium Bradyrhizobium elkanii 587. J Bacteriol 2012; 194:3547-3548. PubMed http://dx.doi.org/10.1128//B.00563-12

5. Kumar S, Vikram S, Raghava GPS. Genome Sequence of the Nitroaromatic CompoundDegrading Bacterium Burkholderia sp. Strain SJ98. J Bacteriol 2012; 194:3286. PubMed http://dx.doi.org/10.1128//B.00497-12

6. Huntley S, Zhang Y, Treuner-Lange A, Kneip S, Sensen CW, Søgaard-Andersen L. Complete Genome Sequence of the Fruiting Myxobacterium Corallococcus coralloides DSM 2259. I Bacteriol 2012; 194:3012-3013. PubMed http://dx.doi.org/10.1128//B.00397-12

7. Mathew MJ, Subramanian G, Nguyen TT, Robert C, Mediannikov O, Fournier PE, Raoult D. Genome Sequence of Diplorickettsia massiliensis, an Emerging lxodes ricinus-Associated Human Pathogen. J Bacterio/ 2012; 194:3287. PubMed http://dx.doi.org/10.1128//B.00448-12 
OW Nelson and GM Garrity

8. Hazen TH, Sahl JW, Redman JC, Morris CR, Daugherty SC, Chibucos MC, Sengamalay NA, Fraser-Liggett CM, Steinsland $\mathrm{H}$, Whittam TS, et al. Draft Genome Sequences of the Diarrheagenic Escherichia coli Collection. J Bacteriol 2012;

194:3026-3027. PubMed http://dx.doi.org/10.1128/JB.00426-12

9. Kim BK, Song GC, Hong GH, Seong WK, Kim SY, Jeong H, Kang SG, Kwon SK, Lee CH, Song JY, et al. Genome Sequence of the Shiga ToxinProducing Escherichia coli Strain NCCP15657. I

Bacteriol 2012; 194:3751-3752. PubMed http://dx.doi.org/10.1128/JB.00654-12

10. Yi H, Cho YJ, Yong D, Chun J. Genome Sequence of Escherichia coli J53, a Reference Strain for Genetic Studies. J Bacteriol 2012; 194:3742-3743. PubMed http://dx.doi.org/10.1128/JB.00641-12

11. Kim BK, Song GC, Hong GH, Seong WK, Kim SY, Jeong H, Kang SG, Kwon SK, Lee CH, Song JY, et al. Genome Sequence of the Shiga ToxinProducing Escherichia coli Strain NCCP15657. I Bacteriol 2012; 194:3751-3752. PubMed http://dx.doi.org/10.1128/JB.00654-12

12. Jeong $\mathrm{H}$, Zhao $F$, Igori D, Oh KH, Kim SY, Kang SG, Kim BK, Kwon SK, Lee CH, Song JY, et al. Genome Sequence of the Hemolytic-Uremic Syndrome-Causing Strain Escherichia coli NCCP15647. J Bacteriol 2012; 194:3747-3748. PubMed http://dx.doi.org/10.1128/JB.00651-12

13. Jeong $\mathrm{H}$, Zhao F, Igori D, Oh KH, Kim SY, Kang SG, Kim BK, Kwon SK, Lee CH, Song JY, et al. Genome Sequence of the Hemolytic-Uremic Syndrome-Causing Strain Escherichia coli NCCP15647. J Bacteriol 2012; 194:3747-3748. PubMed http://dx.doi.org/10.1128/JB.00651-12

14. Zeytun A, Malfatti SA, Vergez LM, Shin M, Garcia E, Chain PSG. Complete Genome Sequence of Francisella philomiragia ATCC 25017. J Bacteriol 2012; 194:3266. PubMed http://dx.doi.org/10.1128/JB.00413-12

15. Kim SM, Cho SJ, Lee SB. Genome Sequence of the Unclassified Marine Gammaproteobacterium BDW918. J Bacteriol 2012; 194:3753-3754. PubMed http://dx.doi.org/10.1128/JB.00678-12

16. Qin QL, Xie BB, Shu YL, Rong JC, Zhao DL, Zhang XY, Chen XL, Zhou BC, Zhang YZ. Genome Sequence of Proteorhodopsin-Containing Sea Ice Bacterium Glaciecola punicea ACAM 611T. J Bacteriol 2012; 194:3267. PubMed http://dx.doi.org/10.1128/JB.00463-12

17. Kawata Y, Kawasaki K, Shigeri Y. Draft Genome Sequence of Halomonas sp. Strain KM-1, a Mod- erately Halophilic Bacterium That Produces the Bioplastic Poly(3-Hydroxybutyrate). J Bacteriol 2012; 194:2738-2739. PubMed http://dx.doi.org/10.1128/JB.00237-12

18. Goto T, Ogura Y, Hirakawa H, Tomida J, Morita Y, Akaike T, Hayashi T, Kawamura Y. Complete Genome Sequence of Helicobacter cinaedi Strain PAGU611, Isolated in a Case of Human Bacteremia. J Bacteriol 2012; 194:3744-3745. PubMed http://dx.doi.org/10.1128/JB.00645-12

19. Behrens W, Bönig T, Suerbaum S, Josenhans C. Genome Sequence of Helicobacter pylori hpEurope Strain N6. J Bacteriol 2012; 194:37253726. PubMed http://dx.doi.org/10.1128/JB.00386-12

20. Kaplan JB, Lo C, Xie G, Johnson SL, Chain PSG, Donnelly R, Kachlany SC, Balashova NV. Genome Sequence of Kingella kingae Septic Arthritis Isolate PYKK081. J Bacteriol 2012; 194:3017. PubMed http://dx.doi.org/10.1128//B.00421-12

21. Hazen TH, Robinson GL, Harris AD, Rasko DA, Johnson JK. Genome Sequence of Klebsiella oxytoca 11492-1, a Nosocomial Isolate Possessing a FOX-5 AmpC $\beta$-Lactamase. / Bacteriol 2012; 194:3028-3029. PubMed http://dx.doi.org/10.1128/JB.00391-12

22. Shin SH, Kim S, Kim JY, Lee S, Um Y, Oh MK, Kim YR, Lee J, Yang KS. Complete Genome Sequence of the 2,3-Butanediol-Producing Klebsiella pneumoniae Strain KCTC 2242. I Bacteriol 2012; 194:2736-2737. PubMed http://dx.doi.org/10.1128/JB.00027-12

23. Guo Y, Cen Z, Zou Y, Fang X, Li T, Wang J, Chang D, Su L, Liu Y, Chen Y, et al. WholeGenome Sequence of Klebsiella pneumonia Strain LCT-KP214. J Bacteriol 2012; 194:3281. PubMed http://dx.doi.org/10.1128/JB.00531-12

24. Grimaud R, Ghiglione JF, Cagnon C, Lauga B, Vaysse PJ, Rodriguez-Blanco A, Mangenot S, Cruveiller S, Barbe V, Duran R, et al. Genome Sequence of the Marine Bacterium Marinobacter hydrocarbonoclasticus SP17, Which Forms Biofilms on Hydrophobic Organic Compounds. J Bacteriol 2012; 194:3539-3540. PubMed http://dx.doi.org/10.1128/JB.00500-12

25. Medrano EG, Bell AA. Genome Sequence of Pantoea sp. Strain Sc 1, an Opportunistic Cotton Pathogen. J Bacteriol 2012; 194:3019. PubMed http://dx.doi.org/10.1128/JB.00450-12

26. Liu W, Yang M, Xu Z, Zheng H, Liang W, Zhou R, Wu B, Chen H. Complete Genome Sequence of Pasteurella multocida HN06, a Toxigenic Strain 
of Serogroup D. J Bacteriol 2012; 194:3292-3293. PubMed http://dx.doi.org/10.1128/JB.00215-12

27. Duquesne K, Prima V, Ji B, Rouy Z, Médigue C, Talla E, Sturgis JN. Draft Genome Sequence of the Purple Photosynthetic Bacterium Phaeospirillum molischianum DSM120, a Particularly Versatile Bacterium. J Bacteriol 2012; 194:3559-3560. PubMed http://dx.doi.org/10.1128/JB.00605-12

28. Clifford RJ, Hang J, Riley MC, Onmus-Leone F, Kuschner RA, Lesho EP, Waterman PE. Complete Genome Sequence of Providencia stuartii Clinical Isolate MRSN 2154. J Bacteriol 2012; 194:37363737. PubMed http://dx.doi.org/10.1128//B.00615-12

29. Xie BB, Shu YL, Qin QL, Rong JC, Zhang XY, Chen XL, Shi M, He HL, Zhou BC, Zhang YZ. Genome Sequences of Type Strains of Seven Species of the Marine Bacterium Pseudoalteromonas. J Bacteriol 2012; 194:2746-2747. PubMed http://dx.doi.org/10.1128/JB.00265-12

30. Fang X, Fang Z, Zhao J, Zou Y, Li T, Wang J, Guo Y, Chang D, Su L, Ni P, et al. Draft Genome Sequence of Pseudomonas aeruginosa Strain ATCC 27853. J Bacteriol 2012; 194:3755. PubMed http://dx.doi.org/10.1128/JB.00690-12

31. Mei Y, Sun Y, He J, Wang Q, Sun Y, Shao W. Genome Sequences of Pseudomonas fragi Strains A22 and B25. J Bacteriol 2012; 194:3276-3277. PubMed http://dx.doi.org/10.1128//B.00488-12

32. Patel HK, da Silva DP, Devescovi G, Maraite H, Paszkiewicz K, Studholme DJ, Venturi V. Draft Genome Sequence of Pseudomonas fuscovaginae, a Broad-Host-Range Pathogen of Plants. J Bacterio/ 2012; 194:2765-2766. PubMed http://dx.doi.org/10.1128/JB.00341-12

33. Tang H, Yu H, Tai C, Huang K, Liu Y, Wang L, Yao Y, Wu G, Xu P. Genome Sequence of a Novel Nicotine-Degrading Strain, Pseudomonas geniculata N1. J Bacteriol 2012; 194:3553-3554. PubMed http://dx.doi.org/10.1128/JB.00625-12

34. Jang SH, Kim J, Kim J, Hong S, Lee C. Genome Sequence of Cold-Adapted Pseudomonas mandelii Strain JR-1. J Bacteriol 2012; 194:3263. PubMed http://dx.doi.org/10.1128/JB.00517-12

35. Mathimaran N, Srivastava R, Wiemken A, Sharma AK, Boller T. Genome Sequences of Two Plant Growth-Promoting Fluorescent Pseudomonas Strains, R62 and R81. J Bacteriol 2012; 194:32723273. PubMed http://dx.doi.org/10.1128/JB.00349-12
36. Sohn KH, Jones JDG, Studholme DJ. Draft Genome Sequence of Pseudomonas syringae Pathovar Syringae Strain FF5, Causal Agent of Stem Tip Dieback Disease on Ornamental Pear. J Bacteriol 2012; 194:3733-3734. PubMed http://dx.doi.org/10.1128/JB.00567-12

37. Martinez RJ, Bruce D, Detter C, Goodwin LA, Han J, Han CS, Held B, Land ML, Mikhailova N, Nolan M, et al. Complete Genome Sequence of Rahnella aquatilis CIP 78.65. J Bacteriol 2012; 194:3020-3021. PubMed http://dx.doi.org/10.1128/JB.00380-12

38. Sentausa E, Karkouri KE, Robert C, Raoult D, Fournier PE. Genome Sequence of Rickettsia Conorii subsp. indica, the Agent of Indian Tick Typhus. J Bacteriol 2012; 194:3288-3289. PubMed http://dx.doi.org/10.1128//B.00462-12

39. Hu P, Lang J, Wawrousek K, Yu J, Maness PC, Chen J. Draft Genome Sequence of Rubrivivax gelatinosus CBS. J Bacteriol 2012; 194:3262. PubMed http://dx.doi.org/10.1128/JB.00515-12

40. Nagashima S, Kamimura A, Shimizu T, Nakamura-Isaki S, Aono E, Sakamoto K, Ichikawa N, Nakazawa H, Sekine M, Yamazaki S, et al. Complete Genome Sequence of Phototrophic Betaproteobacterium Rubrivivax gelatinosus IL144. J Bacteriol 2012; 194:3541-3542. PubMed http://dx.doi.org/10.1128/JB.00511-12

41. Hoffmann M, Zhao S, Luo Y, Li C, Folster JP, Whichard J, Allard MW, Brown EW, McDermott PF. Genome Sequences of Five Salmonella enterica Serovar Heidelberg Isolates Associated with a 2011 Multistate Outbreak in the United States. / Bacteriol 2012; 194:3274-3275. PubMed http://dx.doi.org/10.1128/JB.00419-12

42. Proença DN, Santo CE, Grass G, Morais PV. Draft Genome Sequence of Serratia sp. Strain M24T3, Isolated from Pinewood Disease Nematode Bursaphelenchus xylophilus. I Bacteriol 2012; 194:3764. PubMed http://dx.doi.org/10.1128/JB.00670-12

43. Onodera NT, Ryu J, Durbic T, Nislow C, Archibald JM, Rohde JR. Genome Sequence of Shigella flexneri Serotype 5a Strain M90T Sm. J Bacteriol 2012; 194:3022. PubMed http://dx.doi.org/10.1128/JB.00393-12

44. Lee H, Shin SC, Lee J, Kim SJ, Kim BK, Hong SG, Kim EH, Park H. Genome Sequence of Sphingomonas sp. Strain PAMC 26621, an ArcticLichen-Associated Bacterium Isolated from a Cetraria sp. J Bacteriol 2012; 194:3030. PubMed http://dx.doi.org/10.1128/JB.00395-12 
OW Nelson and GM Garrity

45. Lee J, Shin SC, Kim SJ, Kim BK, Hong SG, Kim EH, Park H, Lee H. Draft Genome Sequence of a Sphingomonas sp., an Endosymbiotic Bacterium Isolated from an Arctic Lichen Umbilicaria sp. J Bacteriol 2012; 194:3010-3011. PubMed http://dx.doi.org/10.1128//B.00360-12

46. Ma Z, Shen X, Hu H, Wang W, Peng H, Xu P, Zhang X. Genome Sequence of Sphingomonas wittichii DP58, the First Reported Phenazine-1Carboxylic Acid-Degrading Strain. [PubMed]. J Bacteriol 2012; 194:3535-3536. PubMed http://dx.doi.org/10.1128//B.00330-12

47. Lira F, Hernández A, Belda E, Sánchez MB, Moya A, Silva FJ, Martínez JL. Whole-Genome Sequence of Stenotrophomonas maltophilia D457, a Clinical Isolate and a Model Strain. [PubMed]. J Bacteriol 2012; 194:3563-3564. PubMed http://dx.doi.org/10.1128//B.00602-12

48. Amaral GRS, Silva BSO, Santos EO, Dias GM, Lopes RM, Edwards RA, Thompson CC, Thompson FL. Genome Sequence of the Bacterioplanktonic, Mixotrophic Vibrio campbellii Strain PEL22A, Isolated in the Abrolhos Bank. J Bacteriol 2012; 194:2759-2760. PubMed http://dx.doi.org/10.1128//B.00377-12

49. Morais LLCS, Garza DR, Loureiro ECB, Nunes KNB, Vellasco RS, da Silva CP, Nunes MRT, Thompson CC, Vicente ACP, Santos EC. Complete Genome Sequence of a SucroseNonfermenting Epidemic Strain of Vibrio cholerae O1 from Brazil. J Bacteriol 2012; 194:2772. PubMed http://dx.doi.org/10.1128//B.00300-12

50. Garza DR, Thompson CC, Loureiro ECB, Dutilh $\mathrm{BE}$, Inada DT, Junior ECS, Cardoso JF, Nunes MRT, de Lima CPS, Silvestre RVD, et al. GenomeWide Study of the Defective Sucrose Fermenter Strain of Vibrio cholerae from the Latin American Cholera Epidemic. PLOS ONE 2012; 7:e37283. PubMed http://dx.doi.org/10.1371/journal.pone.0037283

51. Midha S, Ranjan M, Sharma V, Pinnaka AK, Patil PB. Genome Sequence of Xanthomonas citri pv. mangiferaeindicae Strain LMG 941. J Bacteriol 2012; 194:3031. PubMed http://dx.doi.org/10.1128//B.00433-12

52. Su L, Zhou T, Zhou L, Fang X, Li T, Wang J, Guo Y, Chang D, Wang Y, Li D, et al. Draft Genome Sequence of Bacillus cereus Strain LCT-BC244. I Bacteriol 2012; 194:3549. PubMed http://dx.doi.org/10.1128//B.00580-12

53. Kong M, Kim M, Ryu S. Complete Genome Sequence of Bacillus cereus Bacteriophage PBC1. J
Virol 2012; 86:6379-6380. PubMed

http://dx.doi.org/10.1128/JVI.00706-12

54. Yangtse W, Zhou Y, Lei Y, Qiu Y, Wei X, Ji Z, Qi G, Yong Y, Chen L, Chen S. Genome Sequence of Bacillus licheniformis WX-02. I Bacteriol 2012; 194:3561-3562. PubMed http://dx.doi.org/10.1128//B.00572-12

55. Heggeset TMB, Krog A, Balzer S, Wentzel A, Ellingsen TE, Brautaset T. Genome Sequence of Thermotolerant Bacillus methanolicus. Features and Regulation Related to Methylotrophy and Production of I-lysine and I-glutamate from Methanol [In press]. Appl Environ Microbiol 2012. PubMed http://dx.doi.org/10.1128/AEM.00703-12

56. Song JY, Kim HA, Kim JS, Kim SY, Jeong H, Kang SG, Kim BK, Kwon SK, Lee CH, Yu DS, et al. Genome Sequence of the Plant Growth-Promoting Rhizobacterium Bacillus sp. Strain JS. I Bacteriol 2012; 194:3760-3761. PubMed http://dx.doi.org/10.1128//B.00676-12

57. Kim BK, Chung J, Kim SY, Jeong H, Kang SG, Kwon SK, Lee CH, Song JY, Yu DS, Ryu CM, et al. Genome Sequence of the Leaf-Colonizing Bacterium Bacillus sp. Strain 5B6, Isolated from a Cherry Tree. I Bacteriol 2012; 194:3758-3759. PubMed http://dx.doi.org/10.1128//B.00682-12

58. Jiménez E, Villar-Tajadura MA, Marín M, Fontecha J, Requena T, Arroyo R, Fernández L, Rodríguez JM. Complete Genome Sequence of Bifidobacterium breve CECT 7263, a Strain Isolated from Human Milk. J Bacteriol 2012; 194:37623763. PubMed http://dx.doi.org/10.1128//B.00691-12

59. Kim DS, Jung MY, Sin Y, Kim DW, Paek J, Kim RN, Park IS, Kook JK, Nam SH, Kim A, et al. Genome Sequence of the Anaerobic Bacterium Clostridium arbusti SL206T. J Bacteriol 2012; 194:2758. PubMed http://dx.doi.org/10.1128//B.00368-12

60. Brown SD, Lamed R, Morag E, Borovok I, Shoham Y, Klingeman DM, Johnson CM, Yang Z, Land ML, Utturkar SM, et al. Draft Genome Sequences for Clostridium thermocellum Wild-Type Strain YS and Derived Cellulose AdhesionDefective Mutant Strain AD2. I Bacteriol 2012; 194:3290-3291. PubMed http://dx.doi.org/10.1128//B.00473-12

61. Brown SD, Lamed R, Morag E, Borovok I, Shoham Y, Klingeman DM, Johnson CM, Yang Z, Land ML, Utturkar SM, et al. Draft Genome Sequences for Clostridium thermocellum Wild-Type Strain YS and Derived Cellulose Adhesion- 
Defective Mutant Strain AD2. I Bacteriol 2012;

194:3290-3291. PubMed

http://dx.doi.org/10.1128//B.00473-12

62. Chang D, Zhu Y, Zou Y, Fang X, Li T, Wang J, Guo Y, Su L, Xia J, Yang R, et al. Draft Genome Sequence of Enterococcus faecium Strain LCTEF90. J Bacteriol 2012; 194:3556-3557. PubMed http://dx.doi.org/10.1128//B.00529-12

63. Kergourlay G, Messaoudi S, Dousset X, Prévost H. Genome Sequence of Lactobacillus salivarius SMXD51, a Potential Probiotic Strain Isolated from Chicken Cecum, Showing Anti-

Campylobacter Activity. I Bacteriol 2012;

194:3008-3009. PubMed http://dx.doi.org/10.1128//B.00344-12

64. de Lucena BTL, Silva GGZ, dos Santos BM, Dias GM, Amaral GRS, Moreira APB, Júnior MAM, Dutilh BE, Edwards RA, Balbino V, et al. Genome Sequences of the Ethanol-Tolerant Lactobacillus vini Strains LMG 23202T and JP7.8.9. J Bacteriol 2012; 194:3018. PubMed http://dx.doi.org/10.1128//B.00446-12

65. de Lucena BTL, Silva GGZ, dos Santos BM, Dias GM, Amaral GRS, Moreira APB, Júnior MAM, Dutilh BE, Edwards RA, Balbino V, et al. Genome Sequences of the Ethanol-Tolerant Lactobacillus vini Strains LMG 23202T and JP7.8.9. I Bacteriol 2012; 194:3018. PubMed http://dx.doi.org/10.1128//B.00446-12

66. McMullen PD, Gillaspy AF, Gipson J, Bobo LD, Skiest DJ, Freitag NE. Genome Sequence of Listeria monocytogenes 07PF0776, a Cardiotropic Serovar 4b Strain. J Bacteriol 2012; 194:3552. PubMed http://dx.doi.org/10.1128//B.00616-12

67. Okumura K, Arai R, Okura M, Kirikae T, Takamatsu D, Osaki M, Miyoshi-Akiyama T. Complete Genome Sequence of Melissococcus plutonius DAT561, a Strain That Shows an Unusual Growth Profile and Is Representative of an Endemic Cluster in Japan. J Bacteriol 2012; 194:3014. PubMed http://dx.doi.org/10.1128//B.00437-12

68. Ma M, Wang Z, Li L, Jiang X, Guan D, Cao F, Chen H, Wang X, Shen D, Du B, et al. Complete Genome Sequence of Paenibacillus mucilaginosus 3016, a Bacterium Functional as Microbial Fertilizer. J Bacteriol 2012; 194:2777-2778. PubMed http://dx.doi.org/10.1128//B.00323-12

69. Martinez RJ, Bruce D, Detter C, Goodwin LA, Han J, Han CS, Held B, Land ML, Mikhailova N, Nolan $\mathrm{M}$, et al. Complete Genome Sequence of Rahnella aquatilis CIP 78.65. I Bacteriol 2012;
194:3020-3021. PubMed

http://dx.doi.org/10.1128//B.00380-12

70. Kim DS, Sin Y, Kim DW, Paek J, Kim RN, Jung MY, Park IS, Kim A, Kang A, Park HS, et al. Genome Sequence of the Probiotic Bacterium Sporolactobacillus vineae SL153T. I Bacteriol 2012; 194:3015-3016. PubMed http://dx.doi.org/10.1128//B.00452-12

71. Prabhakara S, Khedkar S, Loganathan RM, Chandana S, Gowda M, Arakere G, Seshasayee ASN. Draft Genome Sequence of Staphylococcus aureus 118 (ST772), a Major Disease Clone from India. / Bacteriol 2012; 194:3727-3728. PubMed http://dx.doi.org/10.1128//B.00480-12

72. Thomas JC, Godfrey PA, Feldgarden M, Robinson DA. Draft Genome Sequences of Staphylococcus aureus Sequence Type 34 (ST34) and ST42 Hybrids. J Bacteriol 2012; 194:2740-2741. PubMed http://dx.doi.org/10.1128//B.00248-12

73. Li G, Hu FZ, Yang X, Cui Y, Yang J, Qu F, Gao GF, Zhang JR. Complete Genome Sequence of Streptococcus pneumoniae Strain ST556, a Multidrug-Resistant Isolate from an Otitis Media Patient. / Bacterio/ 2012; 194:3294-3295. PubMed http://dx.doi.org/10.1128//B.00363-12

74. Chouaia B, Crotti E, Brusetti L, Daffonchio D, Essoussi I, Nouioui I, Sbissi I, Ghodhbane-Gtari F, Gtari M, Vacherie B, et al. Genome Sequence of Blastococcus saxobsidens DD2, a StoneInhabiting Bacterium. J Bacterio/ 2012; 194:27522753. PubMed http://dx.doi.org/10.1128//B.00320-12

75. Sangal V, Tucker NP, Burkovski A, Hoskisson PA. The Draft Genome Sequence of Corynebacterium diphtheriae bv. mitis NCTC 3529 Reveals Significant Diversity between the Primary DiseaseCausing Biovars. J Bacteriol 2012; 194:3269. PubMed http://dx.doi.org/10.1128//B.00503-12

76. Lopes T, Silva A, Thiago R, Carneiro A, Dorella FA, Rocha FS, dos Santos AR, Lima ARJ, Guimarães LC, Barbosa EGV, et al. Complete Genome Sequence of Corynebacterium pseudotuberculosis Strain Cp267, Isolated from a Llama. J Bacteriol 2012; 194:3567-3568. PubMed http://dx.doi.org/10.1128//B.00461-12

77. Brown SD, Palumbo AV, Panikov N, Ariyawansa $\mathrm{T}$, Klingeman DM, Johnson CM, Land ML, Utturkar SM, Epstein SS. Draft Genome Sequence for Microbacterium laevaniformans Strain OR221, a Bacterium Tolerant to Metals, Nitrate, and Low pH. J Bacteriol 2012; 194:3279-3280. PubMed http://dx.doi.org/10.1128//B.00474-12 
OW Nelson and GM Garrity

78. Choi GE, Cho YJ, Koh WJ, Chun J, Cho SN, Shin SJ. Draft Genome Sequence of Mycobacterium abscessus subsp. bolletii BDT. J Bacteriol 2012; 194:2756-2757. PubMed http://dx.doi.org/10.1128//B.00354-12

79. Choo SW, Wong YL, Yusoff AM, Leong ML, Wong G), Ong CS, Ng KP, Ngeow YF. Genome Sequence of the Mycobacterium abscessus Strain M93. I Bacteriol 2012; 194:3278. PubMed http://dx.doi.org/10.1128//B.00492-12

80. Kim BJ, Choi BS, Lim JS, Choi IY, Lee JH, Chun J, Kook YH, Kim BJ. Complete Genome Sequence of Mycobacterium intracellulare Clinical Strain MOTT-02. I Bacteriol 2012; 194:2771. PubMed http://dx.doi.org/10.1128//B.00365-12

81. Kim BJ, Choi BS, Lim JS, Choi IY, Kook YH, Kim BJ. Complete Genome Sequence of Mycobacterium intracellulare Clinical Strain MOTT-64, Belonging to the INT1 Genotype. / Bacteriol 2012; 194:3268. PubMed http://dx.doi.org/10.1128//B.00471-12

82. Kim BJ, Choi BS, Lim JS, Choi IY, Lee JH, Chun J, Kook YH, Kim BJ. Complete Genome Sequence of Mycobacterium intracellulare Strain ATCC 13950T. J Bacteriol 2012; 194:2750. PubMed http://dx.doi.org/10.1128//B.00295-12

83. Abdallah AM, Rashid M, Adroub SA, Arnoux M, Ali S, van Soolingen D, Bitter W, Pain A. Complete Genome Sequence of Mycobacterium phlei Type Strain RIVM601174. I Bacteriol 2012; 194:3284-3285. PubMed http://dx.doi.org/10.1128//B.00485-12

84. Miyoshi-Akiyama T, Matsumura K, Iwai $\mathrm{H}$, Funatogawa K, Kirikae T. Complete Annotated Genome Sequence of Mycobacterium tuberculosis Erdman. J Bacteriol 2012; 194:2770. PubMed http://dx.doi.org/10.1128//B.00353-12

85. Abdallah AM, Rashid M, Adroub SA, Elabdalaoui $\mathrm{H}$, Ali S, van Soolingen D, Bitter W, Pain A. Complete Genome Sequence of Mycobacterium xenopi Type Strain RIVM700367. I Bacteriol 2012; 194:3282-3283. PubMed http://dx.doi.org/10.1128//B.00482-12

86. Vera-Cabrera L, Ortiz-Lopez R, ElizondoGonzalez R, Perez-Maya AA, Ocampo-Candiani J. Complete Genome Sequence of Nocardia brasiliensis HUJEG-1. J Bacteriol 2012; 194:27612762. PubMed http://dx.doi.org/10.1128//B.00210-12

87. McDowell A, Hunyadkürti J, Horváth B, Vörös A, Barnard E, Patrick S, Nagy I. Draft Genome Sequence of an Antibiotic-Resistant
Propionibacterium acnes Strain, PRP-38, from the Novel Type IC Cluster. I Bacteriol 2012;

194:3260-3261. PubMed http://dx.doi.org/10.1128//B.00479-12

88. Kang I, Lee K, Yang SJ, Choi A, Kang D, Lee YK, Cho JC. Genome Sequence of "Candidatus Aquiluna" sp. Strain IMCC13023, a Marine Member of the Actinobacteria Isolated from an Arctic Fjord. J Bacteriol 2012; 194:3550-3551. PubMed http://dx.doi.org/10.1128//B.00586-12

89. Zhang Y, Qin F, Qiao J, Li G, Shen C, Huang T, Hu Z. Draft Genome Sequence of Rhodococcus sp. Strain P14, a Biodegrader of High-MolecularWeight Polycyclic Aromatic Hydrocarbons. J Bacteriol 2012; 194:3546. PubMed http://dx.doi.org/10.1128//B.00555-12

90. Vikram S, Kumar S, Subramanian S, Raghava GPS. Draft Genome Sequence of the NitrophenolDegrading Actinomycete Rhodococcus imtechensis RKJ300. J Bacteriol 2012; 194:3543. PubMed http://dx.doi.org/10.1128//B.00532-12

91. Aikawa C, Furukawa N, Watanabe T, Minegishi K, Furukawa A, Eishi Y, Oshima K, Kurokawa K, Hattori M, Nakano K, et al. Complete Genome Sequence of the Serotype k Streptococcus mutans Strain LJ23. I Bacteriol 2012; 194:2754-2755. PubMed http://dx.doi.org/10.1128//B.00350-12

92. Kirby R, Sangal V, Tucker NP, ZakrzewskaCzerwińska J, Wierzbicka K, Herron PR, Chu CJ, Chandra G, Fahal AH, Goodfellow M, et al. Draft Genome Sequence of the Human Pathogen Streptomyces somaliensis, a Significant Cause of Actinomycetoma. J Bacteriol 2012; 194:35443545. PubMed http://dx.doi.org/10.1128//B.00534-12

93. Guo M, Han X, Jin T, Zhou L, Yang J, Li Z, Chen J, Geng B, Zou Y, Wan D, et al. Genome Sequences of Three Species in the Family Planctomycetaceae. I Bacteriol 2012; 194:37403741. PubMed http://dx.doi.org/10.1128//B.00639-12

94. Elbir H, Gimenez G, Robert C, Bergström S, Cutler S, Raoult D, Drancourt M. Complete Genome Sequence of Borrelia crocidurae. I Bacteriol 2012; 194:3723-3724. PubMed http://dx.doi.org/10.1128//B.00118-12

95. Lin B, Lu G, Li S, Hu Z, Chen H. Draft Genome Sequence of the Novel Agarolytic Bacterium Aquimarina agarilytica ZC1. J Bacteriol 2012; 194:2769. PubMed http://dx.doi.org/10.1128//B.00311-12 
96. Filippini M, Qi W, Blom J, Goesmann A, Smits THM, Bagheri HC. Genome Sequence of Fibrella aestuarina BUZ 2T, a Filamentous Marine Bacterium. / Bacterio/ 2012; 194:3555. PubMed http://dx.doi.org/10.1128/JB.00550-12

97. Tekedar HC, Karsi A, Gillaspy AF, Dyer DW, Benton NR, Zaitshik J, Vamenta S, Banes MM, Gülsoy N, Aboko-Cole M, et al. Genome Sequence of the Fish Pathogen Flavobacterium columnare ATCC 49512. J Bacteriol 2012;

194:2763-2764. PubMed http://dx.doi.org/10.1128//B.00281-12

98. Barbier P, Houel A, Loux V, Poulain J, Bernardet JF, Touchon M, Duchaud E. Complete Genome Sequence of Flavobacterium indicum GPSTA1009T, Isolated from Warm Spring Water. J Bacteriol 2012; 194:3024-3025. PubMed http://dx.doi.org/10.1128//B.00420-12

99. Nam YD, Lee HW, Lee M, Yim KJ, Kim KN, Roh SW, Kim D. Draft Genome Sequence of Gillisia sp. Strain CBA3202, a Novel Member of the Genus Gillisia, Which Belongs to the Family Flavobacteriaceae. J Bacteriol 2012; 194:3739. PubMed http://dx.doi.org/10.1128//B.00631-12

100. Kumar S, Vikram S, Subramanian S, Raghava GPS, Pinnaka AK. Genome Sequence of the Halotolerant Bacterium Imtechella halotolerans K1T. J Bacteriol 2012; 194:3731. PubMed http://dx.doi.org/10.1128//B.00506-12

101. Kim DS, Paek J, Shin JH, Kim DW, Jung MY, Kim $\mathrm{RN}$, Sin Y, Kook JK, Nam SH, Kim A, et al. Genome Sequence of Myroides injenensis M090166T, Isolated from Clinical Specimens. J Bacteriol 2012; 194:2748-2749. PubMed http://dx.doi.org/10.1128//B.00276-12
102. Kumar S, Subramanian S, Raghava GPS, Pinnaka AK. Genome Sequence of the Marine Bacterium Marinilabilia salmonicolor JCM 21150T. J

Bacteriol 2012; 194:3746. PubMed http://dx.doi.org/10.1128//B.00649-12

103. Lee M, Roh SW, Lee HW, Yim KJ, Kim KN, Bae JW, Choi KS, Jeon YJ, Jung WK, Kang H, et al. Draft Genome Sequence of Pedobacter agri PB92T, Which Belongs to the Family Sphingobacteriaceae. I Bacteriol 2012; 194:3738. PubMed http://dx.doi.org/10.1128//B.00627-12

104. Wang X, Zhu D, Wang M, Cheng A, Jia R, Zhou Y, Chen Z, Luo Q, Liu F, Wang Y, et al. Complete Genome Sequence of Riemerella anatipestifer Reference Strain. J Bacteriol 2012; 194:32703271. PubMed http://dx.doi.org/10.1128//B.00366-12

105. Khadem AF, Wieczorek AS, Pol A, Vuilleumier S, Harhangi HR, Dunfield PF, Kalyuzhnaya MG, Murrell JC, Francoijs KJ, Stunnenberg HG, et al. Draft Genome Sequence of the VolcanoInhabiting Thermoacidophilic Methanotroph Methylacidiphilum fumariolicum Strain SolV. J Bacteriol 2012; 194:3729-3730. PubMed http://dx.doi.org/10.1128//B.00501-12

106. Isanapong J, Goodwin L, Bruce D, Chen A, Detter C, Han J, Han CS, Held B, Huntemann M, Ivanova N, et al. High-Quality Draft Genome Sequence of the Opitutaceae Bacterium Strain TAV1, a Symbiont of the Wood-Feeding Termite Reticulitermes flavipes. I Bacteriol 2012; 194:2744-2745. PubMed http://dx.doi.org/10.1128//B.00264-12 